\title{
Parameters adaptive backstepping control of PMSM using DTC method
}

\author{
Xiaoming Zheng*, Lei Xue, Peng Wang, Jia Li and Zeyuan Shen \\ Planning Review Center, Economic and Electrical Research Institute of Shanxi Electrical Power Company of SGCC, Taiyuan, Shanxi, \\ 030002, China
}

\begin{abstract}
The torque and flux ripple of traditional DTC control of PMSM is large, and due to the lack of adaptive control system, the change of the system parameters has a great impact on the steady and transient control performance of the motor speed. Based on the traditional DTC control algorithm, a parameter identification adaptive backstepping DTC control algorithm is proposed in this paper. Because the backstepping controller is used to replace PI controller and flux hysteresis controller, the steady-state and transient performance of the system is obviously improved. At the same time, a parameter identification adaptive controller is designed, which can effectively suppress the influence of parameter changes on the speed control performance. The simulation results verify the correctness and effectiveness of the control algorithm. The control algorithm can significantly improve the steady-state and transient performance of the system under the condition of parameter mutation.
\end{abstract}

\section{Introduction}

The traditional direct torque control (DTC) of permanent magnet synchronous motor(PMSM $)^{[1,2]}$ has the advantages of less parameter dependence and faster response speed. However, due to the hysteresis controller used in the torque and flux control, the flux and torque ripple is too large $\mathrm{e}^{[3]}$. At the same time, the PI controller is used in the speed loop, resulting in a slow speed response. The backstepping control is a kind of nonlinear system control strategy and the decoupling and order reduction of control system are realized by Lyapunov function. In reference [4], a multi-stage hysteresis controller is proposed to improve the torque and flux ripple of traditional DTC. In reference [5], a novel sliding mode controller is designed, which combined with space vector modulation, can effectively reduce the torque and flux ripple of traditional DTC. Based on the backstepping control algorithm ${ }^{[6-7]}$, reference [8] proposed a control strategy based on space vector modulation, which has the same response speed as the traditional DTC, while significantly reducing the torque and flux ripple.

However, the above control methods do not consider the system response optimization under the condition of system parameter mutation. In high precision control system, the influence of these parameters should be considered. In this paper, the backstepping control of PMSM using DTC method is proposed by combining the backstepping control with adaptive control algorithm. This method can not only improve the performance of traditional DTC, but also suppress the influence of parameter deviation and improve the robustness of the system.

\section{System Description}

\subsection{Mathematical model of PMSM}

$$
\begin{gathered}
\varphi_{\mu}=\varphi_{v}^{2}+\varphi_{o}^{2}, \quad l=\frac{1}{J}, \quad \kappa=\frac{B}{J}, \quad v=\frac{1}{L_{d}}, \quad o=\frac{L_{d}}{L_{d}-L_{q}} \\
T_{e}=1.5 n_{p}\left(\varphi_{v} i_{o}+\varphi_{o} i_{v}\right), \quad \frac{\mathrm{d} \omega}{\mathrm{d} t}=\eta n_{p}\left(T_{e}-T_{L}\right)-\kappa \omega \\
\left\{\begin{array}{l}
\frac{d i_{v}}{d t}=-v R i_{v}-o \omega i_{o}-v E_{v}+v u_{v} \\
\frac{d i_{o}}{d t}=-o \omega i_{v}-v \mathrm{Ri}_{o}-v E_{o}+v u_{o}
\end{array}\right.
\end{gathered}
$$

Where $\varphi_{\mu}$ is the stator flux linkage, $\varphi_{v}, \varphi_{o}$ are the $\alpha$ axis and $\beta$ axis flux linkage, $J$ is the intertia, $B$ is the viscous friction coefficient, $i_{v}$ and $i_{o}$ are the components of stator current in $\alpha$ axis and $\beta$ axis, $R$ is the phase resistance, $\omega$ is mechanical angular velocity, $L_{V}$ and $L_{o}$ are the equivalent inductances in $d$ axis and $q$ axis, $E_{v}$ and $E_{o}$ are the EMF in $d$ axis and $q$ axis, $T_{\mathrm{e}}$ is the electromagnetic torque, $n_{\mathrm{p}}$ is the number of rotor pole pairs. 


\subsection{Control strategy design}

$$
\begin{aligned}
& \xi_{\omega}=\omega-\omega_{r e f}, \xi_{T}=T_{e}-T_{r e f}, \xi_{\varphi}=\varphi_{s}-\varphi_{r e f} \\
& \eta_{1}=\frac{1}{2} \xi_{\omega}^{2}+\frac{1}{2} \xi_{T}^{2}+\frac{1}{2} \xi_{\varphi}^{2} \\
& T_{L}=T_{L}^{*}-\Delta T_{L}, R=R^{*}-\Delta R \\
& u_{v}^{*}=\frac{2 \varphi_{o}}{3 n_{P}\left[\varphi_{o}\left(i_{o}-\varphi_{o} v\right)+\varphi_{v}\left(i_{v}-\varphi_{o} v\right)\right]} \cdot\left[1.5 n_{p}\left(\varphi_{v} v i_{o}+\varphi_{o} v i_{v}\right) R^{*}-1.5 n_{p}\left(\omega o i_{v}-v E_{o}\right) \varphi_{v}\right. \\
& -l n_{p} \xi_{\omega}-1.5 n_{p}\left(\varphi_{o} v-i_{v}\right)\left(\frac{R^{*} \varphi_{v} i_{v}}{\varphi_{o}}+R^{*} i_{o}-\frac{k_{\varphi} \xi_{\varphi}}{2 \varphi_{o}}\right)-1.5 n_{P}\left(\omega o i_{o}+v E_{v}\right)+\left(\kappa-k_{\omega}\right) T_{e} \\
& \left.+\left(\kappa-k_{\omega}\right) T_{L}^{*}+\frac{\kappa \omega\left(\kappa-k_{\omega}\right)}{l n_{p}}+\frac{\ddot{\omega}^{*}}{l n_{p}}+\frac{k_{\omega} \dot{\omega}^{*}}{l n_{p}}+\dot{T}_{L}^{*}-k_{T} \xi_{T}\right] \\
& u_{o}^{*}=\frac{2 \varphi_{v}}{3 n_{P}\left[\varphi_{o}\left(\varphi_{o} v-i_{o}\right)+\varphi_{v}\left(\varphi_{o} v-i_{v}\right)\right]} \cdot\left[1.5 n_{p}\left(\varphi_{v} v i_{o}+\varphi_{o} v i_{v}\right) R^{*}-1.5 n_{p}\left(\omega o i_{v}-v E_{o}\right) \varphi_{v}\right. \\
& -i n_{p} \xi_{\omega}-1.5 n_{p}\left(i_{o}-\varphi_{o} v\right)\left(\frac{R^{*} \varphi_{v} i_{v}}{\varphi_{o}}+R^{*} i_{v}-\frac{k_{\varphi} \xi_{\varphi}}{2 \varphi_{v}}\right)-1.5 n_{P}\left(\omega o i_{o}+v E_{v}\right) \varphi_{\beta}+\left(\kappa-k_{\omega}\right) T_{e} \\
& \left.+\left(\kappa-k_{\omega}\right) T_{L}^{*}+\frac{\kappa \omega\left(\kappa-k_{\omega}\right)}{l n_{p}}+\frac{\ddot{\omega}^{*}}{l n_{p}}+\frac{k_{\omega} \dot{\omega}^{*}}{l n_{p}}+\dot{T}_{L}^{*}-k_{T} \xi_{T}\right]
\end{aligned}
$$
estimations errors. The $\alpha$ and $\beta$ axis voltage can be chosen

By substitution of (6), (7) and (8) into the derivative of (5), we can obtain

$$
\begin{aligned}
\eta_{2} & =-k_{\omega} \xi_{\omega}^{2}-k_{T} \xi_{T}^{2}-k_{\varphi} \xi_{\varphi}^{2}+i n_{p} e_{\omega} \Delta T_{L}+\left(\kappa-k_{\omega}\right) \Delta T_{L} \\
& +1.5 n_{p}\left(\varphi_{v} v i_{o}+\varphi_{o} v i_{v}\right) \Delta R+\xi_{\varphi}\left(2 \Delta R \varphi_{\nu} i_{v}+2 \Delta R \varphi_{o} i_{o}\right)
\end{aligned}
$$

Lyapunov function is chosen as follows

$$
\eta_{2}=\eta_{1}+\frac{\Delta R^{2}}{2 \lambda_{1}}+\frac{\Delta T_{L}^{2}}{2 \lambda_{2}}
$$

Where $\lambda_{1}, \lambda_{2}$ represents positive adaptive gains. Adaptive law is obtained:

$$
\Delta \dot{T}_{L}=\lambda_{2}\left(k_{\omega}-\kappa-l n_{p} \xi_{\omega}\right)
$$

$$
\Delta \dot{R}=-1.5 n_{p}\left(\varphi_{v} v i_{o}+\varphi_{o} v i_{v}\right)-2 \xi_{\varphi} \lambda_{1}\left(\varphi_{v} i_{v}+\varphi_{o} i_{o}\right)
$$

By substitution of (11), (12) into the derivative of (10), we can obtain

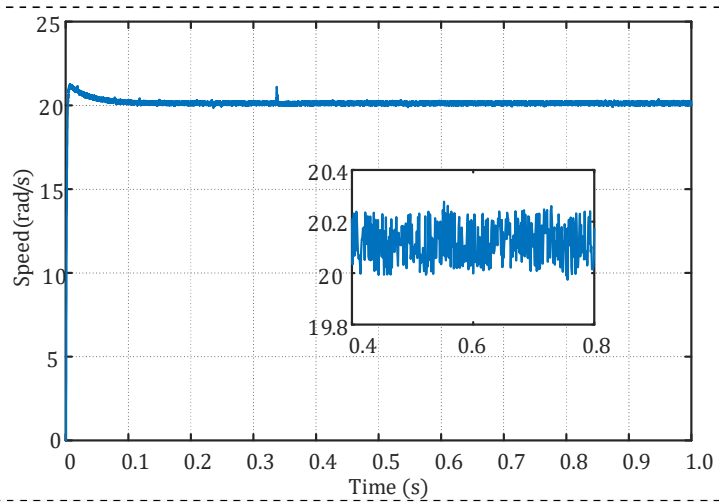

Fig 1. Speed wave of the general DTC algorithm.

$$
\begin{aligned}
& \frac{d \eta_{2}}{d t}=-k_{\omega} \xi_{\omega}^{2}-k_{T} \xi_{T}^{2}-k_{\varphi} \xi_{\varphi}^{2} \leq 0 \\
& \lim _{t \rightarrow \infty} \xi_{\omega}=\lim _{t \rightarrow \infty} \xi_{T}=\lim _{t \rightarrow \infty} \xi_{\varphi}=0
\end{aligned}
$$

Selection of suitable controller gains $k_{\omega}, k_{T}, k_{\varphi}$ and adaptive law gains $r_{1}, r_{2}$ can ensure the tracking error signals of system are asymptotically stable. That is to say, the control method proposed in this paper can quickly track the given reference signals and restrain the influence of outside interference at the same time.

\section{Simulation Results}

The controller gains value are $k_{\omega}=0.02, k_{T}=100, k_{\varphi}$ $=1000$. The adaptive identification parameters are $t_{1}=$ 4.12, $t_{2}=5.58$. The results of comparative experiments with reference speed $\omega=20 \mathrm{rad} / \mathrm{s}$ and load torque $T_{L}=28 \mathrm{~N} \cdot \mathrm{m}$ are shown below.

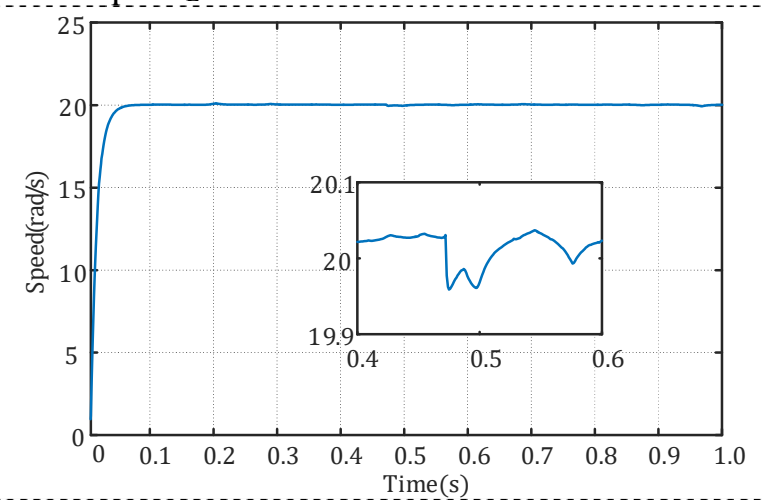

Fig 2. Speed wave of the algorithm in this paper. 


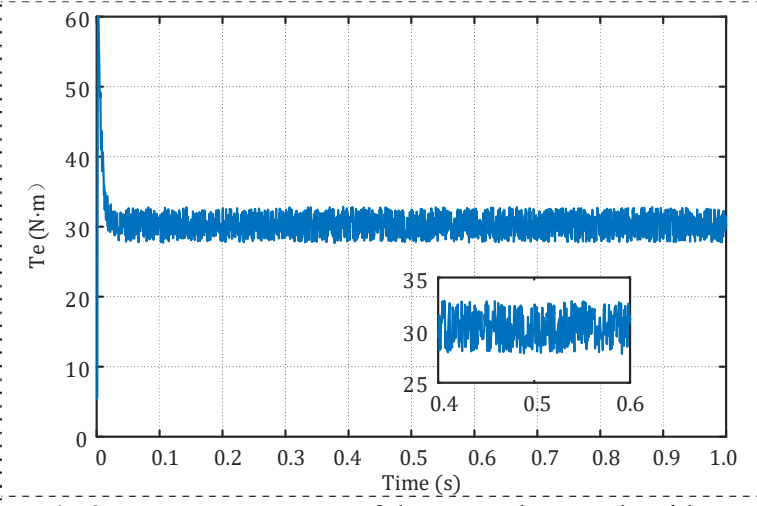

Fig 3. Torque wave of the general DTC algorithm.

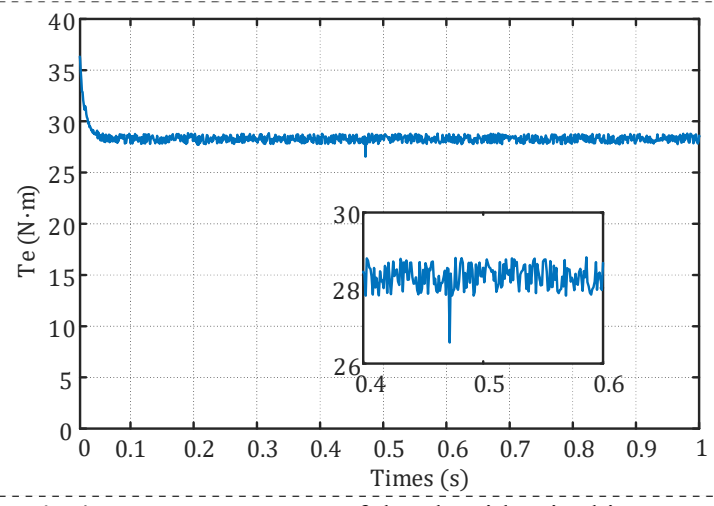

Fig 4. Torque wave of the algorithm in this paper.
From the above comparison, it can be seen that through the algorithm optimization, the speed and torque

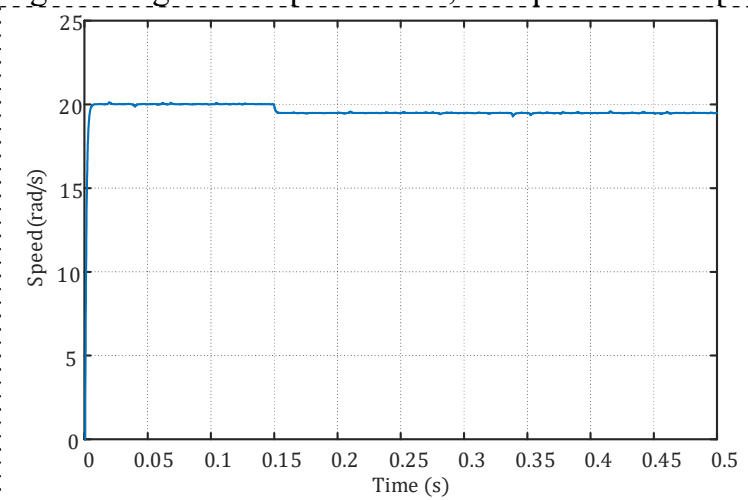

(a) ripple are significantly improved, and the response time is also significantly improved.

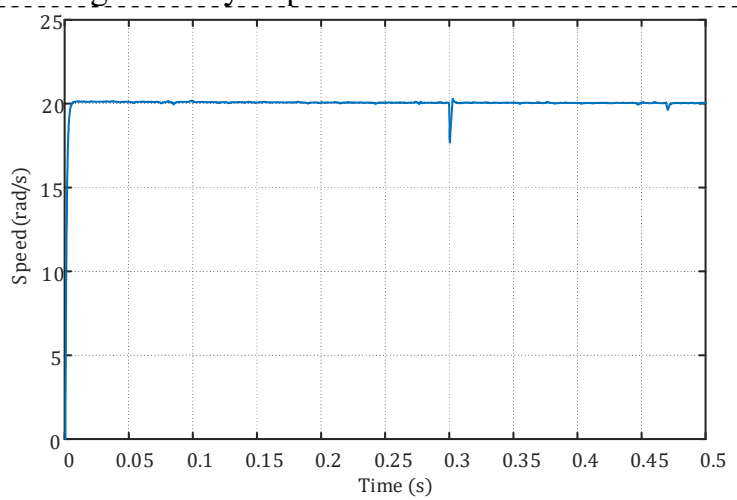

(b)

Fig 5. Speed wave of backstepping the general DTC without adaptive law

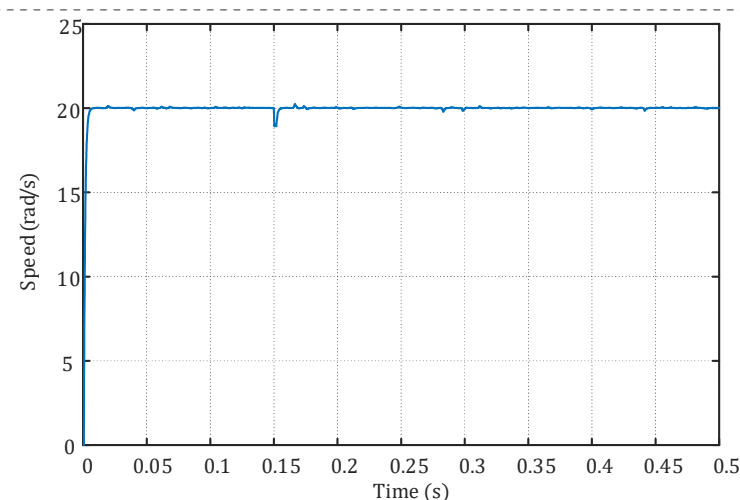

(a)

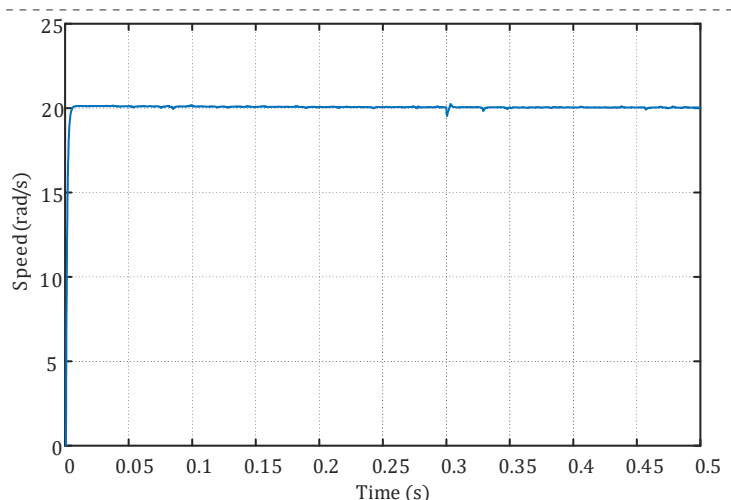

(b)

Fig 6. Speed wave of the algorithm in this paper with adaptive law

In order to verify the suppression of stator resistance and load mutation, comparative experiments are carried out. The stator resistance $R s$ is increased to $0.4 \Omega$ in $0.15 \mathrm{~s}$, and the load torque $T_{L}$ is increased to $42 \mathrm{~N} \cdot \mathrm{m}$ in $0.3 \mathrm{~s}$. The speed change of PMSM is analyzed under the condition of parameter mutation.

The simulation results show that in the traditional algorithm, the speed deviates from the reference value about $1 \mathrm{rad} / \mathrm{s}$ in $0.15 \mathrm{~s}$, and there is an obvious jitter peak at $0.3 \mathrm{~s}$. The control algorithm in this paper only has a small amplitude peak at $0.15 \mathrm{~s}$ and $0.3 \mathrm{~s}$, which can quickly recover to the reference value.

\section{Conclusion}

The traditional DTC control has large torque and speed ripple, and the steady state value of speed will shift when the parameter changes suddenly, which will affect the accuracy and robustness of the control system. The control algorithm proposed in this paper can solve the above problems. From the simulation results, it can be seen that the proposed control algorithm not only significantly reduces the torque and speed ripple, but also significantly shortens the response time. At the same time, there is no 
static error in the speed tracking under the condition of parameter drift, which significantly improves the accuracy and robustness of the control system. The proposed control algorithm in this paper is of great significance to the precise control of the PMSM.

\section{References}

1. Zhong, L, Rahman $\mathrm{M} \mathrm{F}, \mathrm{Hu} \mathrm{W}$ Y, Lim KW. Analysis of direct torque control in permanent magnet synchronous motor drives. IEEE Transactions on Power Electronics, 1993, 12(3): 528-536.

2. Rahman M F, Zhong L, Haque M E, Rahman MA. A direct torque-controlled interior permanentmagnet synchronous motor drive without a speed sensor. Information and the modern corporation/ MIT Press, 2009, 16(2): 137-147.

3. Dwivedi, S, and B. Singh. Vector Control vs Direct Torque Control comparative evaluation for PMSM drive. Joint International Conference on Power Electronics, Drives and Energy Systems IEEE, 2010, 18(8): 1-8.

4. Xiao Meng, SHI Tingna, Wang Zhiqiang, et al. Direct torque control for permanent magnet synchronous motor with multilevel hysteresis controller [J]. Proceedings of the CSEE, 2017, 37(14):4201-4211.

5. Song Zhengguang, Xia Changliang, Wang Zhiqiang, et al. Direct torque control for permanent magnet synchronous motor using super twisting algorithm [J]. Transactions of China Electrotechnical Society, 2017, 32(15):89-99.

6. Yu Yang, Guo Xudong, Zheng Xiaoming, Mi Zengqiang. Backstepping control based maximum torque per ampere control of permanent magnet synchronous motor for mechanical elastic energy storage [J]. Transactions of China Electrotechnical Society, 2017, 32(22): 82-90.

7. Liu Xiaohui, Liu Xiaoping, Liu Hesheng, et al. Chaos Synchronization Control in Permanent Magnet Synchronous Motor Based on Backstepping $[\mathrm{J}]$. Electrical Measurement \& Instrumentation, 2012, 49(12): 37-40.

8. Xu Yanping, Lei Yazhou, Ma Lingzhi, et al. A novel direct torque control of permanent magnet synchronous motors based backstepping control [J]. Transactions of China Electrotechnical Society, 2015, 30(10):83-89. 